IMACOLADA Marino TOZO ${ }^{1}$

José CÁsSIO MORAEs ${ }^{2}$

SONIA MARIA ROLIM LIMA ${ }^{3}$

Nelson GONÇALVES ${ }^{4}$

ANTONIO PEDRo Flores AugE ${ }^{5}$

Lia Mara Rossi ${ }^{6}$

TSUTOMU AOKI ${ }^{7}$

Artigo original

Palavras-chave

Sexualidade

Histerectomia/métodos

Leiomioma/cirurgia

Disfunção sexual fisiológica

Keywords

Sexuality

Hysterectomy/methods

Leiomyoma/surgery

Sexual dysfunction, physiological

\section{Avaliação da sexualidade em mulheres submetidas à histerectomia para tratamento do leiomioma uterino}

\author{
Sexuality evaluation in women submitted to bysterectomy for the \\ treatment of uterine leiomyoma
}

\section{Resumo}

OBJETIVO: avaliar o impacto da histerectomia sobre a sexualidade de mulheres portadoras de leiomioma uterino. MÉTODOS: estudo prospectivo, no qual foram incluídas 33 mulheres sexualmente ativas, com idade de 35 a 50 anos, experiência orgástica e parceiro fixo capacitado para o coito. Todas as mulheres foram submetidas a dois instrumentos para avaliação da sexualidade: Quociente Sexual - Versão Feminina (QS-F) e Inventário de Satisfação Sexual Versão Feminina (GRISS). Os mesmos instrumentos foram aplicados pelo mesmo examinador antes da histerectomia e seis meses após o procedimento. RESULTADOS: o QS-F apontou que 39,4\% das pacientes apresentaram piora no relacionamento sexual, apesar de não ter sido encontrada associação entre os resultados obtidos no QS-F antes e depois da histerectomia $\left(\chi^{2}=10,6\right.$; grau de liberdade=12; $\left.p=0,05\right)$. Os escores médios obtidos após a aplicação do questionário de GRISS mostraram piora significante nos parâmetros "satisfação sexual" (p=0,03); "expressão da sensualidade feminina" ( $p=0,01)$; "vaginismo/dispareunia" $(p=0,02)$ e "anorgasmia" $(p=0,04)$. CONCLUSÕES: a histerectomia parece impactar negativamente a vida sexual das mulheres, sendo referida pela diminuição do desejo, da excitação e da capacidade orgásmica.

\section{Abstract}

PURPOSE: to evaluate the impact of hysterectomy on the sexuality of women with uterine leiomyoma. METHODS: prospective study including 33 sexually active women, with ages from 35 to 50 years old, with orgasmic experience and with a fit stable partner. All the women were submitted to two instruments for the evaluation or their sexuality: Sexual Quotient - Female Version (SQF) and Sexual Satisfaction Inventory - Female Version (SSIF). Both instruments were applied by the same examiner, before and six months after the hysterectomy. RESULTS: the SQF has shown that 39.4\% of the patients presented deterioration in the sexual intercourse, even though there has not been found an association between the SQF results before and after hysterectomy $\left(\chi^{2}=0.6\right.$; degree of freedom $\left.=12 ; p=0.05\right)$. The mean scores obtained after the application of the SSIF have shown significant deterioration in the following parameters: sexual satisfaction $(p=0.03)$; expression of feminine sensuality $(p=0.01)$; vaginismus/dyspareunia $(p=0.02)$ and anorgasmia $(p=0.04)$. CONCLUSIONS: it seems that hysterectomy has a negative impact on women's sexual life, with reports of decreased libido, arousal and orgasmic capacity.
Correspondência:

Imacolada Marino Tozo Rua Dr Cesário Mota Jr, 112 - Vila Buarque CEP 01221020 - São Paulo (SP), Brasil E-mail: tinamarino@terra.com.br

Recebido

23/10/08

Aceito com modificacões 22/9/09
Departamento de Obstetrícia e Ginecologia da Faculdade de Ciências Médicas da Santa Casa de São Paulo - FCMSCSP - São Paulo (SP), Brasil.

'Psicóloga; Pós-graduanda (Doutorado) da Faculdade de Ciências Médicas da Santa Casa de São Paulo - FCMSCSP - São Paulo (SP), Brasil.

2 Professor Adjunto do Departamento de Medicina Social da Faculdade de Ciências Médicas da Santa Casa de São Paulo FCMSCSP - São Paulo (SP), Brasil.

3 Professora Adjunta do Departamento de Obstetrícia e Ginecologia da Faculdade de Ciências Médicas da Santa Casa de São Paulo - FCMSCSP - São Paulo (SP), Brasil.

${ }^{4}$ Instrutor de Ensino do Departamento de Obstetrícia e Ginecologia da Faculdade de Ciências Médicas da Santa Casa de São Paulo - FCMSCSP - São Paulo (SP), Brasil

5 Professor Adjunto do Departamento de Obstetrícia e Ginecologia da Faculdade de Ciências Médicas da Santa Casa de São Paulo - FCMSCSP - São Paulo (SP), Brasil.

Pós-graduanda (Doutorado) da Faculdade de Ciências Médicas da Santa Casa de São Paulo - FCMSCSP - São Paulo (SP), Brasil; Coordenadora da Secretaria de Apoio à Pesquisa da Faculdade de Ciências Médicas da Santa Casa de São Paulo - FCMSCSP São Paulo (SP), Brasil; Professora-Assistente da Faculdade de Medicina de Jundiaí - FMJ - Jundiaí (SP), Brasil.

7 Professor Adjunto e Chefe do Departamento de Obstetrícia e Ginecologia da Faculdade de Ciências Médicas da Santa Casa de São Paulo - FCMSCSP - São Paulo (SP), Brasil. 


\section{Introdução}

Estima-se que entre 20 a $50 \%$ das mulheres entre a terceira e a quinta décadas da vida sejam portadoras de tumores uterinos benignos. Esses incidem principalmente em mulheres da raça negra, aparecendo numa proporção de três a nove vezes em relação à raça branca. Podem ser classificados, independentemente da sintomatologia, de acordo com a camada uterina em que se desenvolvem em subserosos, intramurais ou submucosos ${ }^{1,2}$. A presença de leiomioma uterino parece ser a maior causa para a indicação da histerectomia que visa além da resolução definitiva do leiomioma ${ }^{3}$.

Além de suas funções biológicas, o útero associa-se ao conceito de feminilidade por relacionar-se ao papel reprodutor da mulher e à sua vida sexual. Do ponto de vista psicológico, especula-se que a retirada do útero pode causar prejuízos na qualidade da vida sexual da mulher, em suas condições emocionais e na qualidade do relacionamento estabelecido com o parceiro ${ }^{4}$. No entanto, estudos mostram que as mulheres só se dão conta da importância do útero como garantia de ser mulher após a sua retirada ${ }^{5-7}$. Parece plausível, nesse contexto, que a necessidade de realizar uma cirurgia para a retirada desse órgão pode, em muitos casos, provocar emoções conflitivas, traumáticas, de insegurança e ansiedade, gerando mudanças importantes nos padrões e no desejo sexuais.

Associadas aos aspectos emocionais, ocorrem também modificações anatômicas na pelve, que podem levar a alteração do tamanho e/ou do formato dos órgãos genitais, dificuldade de penetração vaginal, dispareunia, interrupção dos suportes anatômicos da resposta sexual, rebaixamento do impulso sexual e do grau de atratividade por redução de níveis hormonais circulantes, decorrentes de alterações circulatórias, ocasionando, em última instância, disfunções sexuais. Outros autores, entretanto, apontam para a histerectomia como fator causal para o aumento da frequência de dispareunia mediana e profunda pelo encurtamento da vagina, diminuição da libido e menor frequência de orgasmos após a penetração ${ }^{8-11}$.

Acredita-se que mulheres submetidas à histerectomia podem apresentar alterações no autoconceito, sintomas depressivos e psicossomáticos, dificuldade de relacionamento sexual e conjugal, devido a associações psicológicas feitas entre a procriação e a sexualidade. No entanto, as reais consequências da realização da histerectomia sobre a qualidade da vida sexual posterior à cirurgia são controversas e dependem diretamente da intensidade dos sintomas précirúrgicos, das condições emocionais e da qualidade do relacionamento estabelecido com o parceiro. É consenso que a histerectomia diminui sobremaneira o desconforto causado pelos leiomiomas, mas pouco tem sido publicado a respeito dos impactos emocional da histerectomia sobre a mulher. Estudos têm apresentado resultados controversos sobre as consequências da retirada do útero, não somente na esfera emocional, mas, sobretudo, na função sexual feminina. Não apontam, no entanto, de forma conclusiva, os efeitos pós-cirúrgicos sobre a qualidade de vida sexual da mulher, deixando lacunas acerca da real interferência emocional do procedimento ${ }^{7,12,13}$.

Com base nessas informações, este estudo teve por objetivo avaliar a sexualidade em mulheres submetidas à histerectomia para tratamento de leiomioma uterino e comparar a resposta sexual feminina prévia à histerectomia àquela obtida seis meses após a cirurgia - tempo suficiente para a retomada da atividade sexual.

\section{Métodos}

Foram selecionadas 79 mulheres com diagnóstico de leiomioma uterino e indicação para histerectomia total abdominal. O trabalho foi desenvolvido nos Ambulatórios Geral de Sexologia e na Enfermaria do Departamento de Obstetrícia e Ginecologia (DOGi) da Faculdade de Ciências Médicas da Santa Casa de São Paulo da Irmandade da Santa Casa de Misericórdia de São Paulo (ISCMSP) no período de janeiro a setembro de 2007. O projeto foi aprovado pelo Comitê de Ética em Pesquisa da ISCMSP (487/06), e todas as mulheres recrutadas para o estudo assinaram o termo de consentimento livre e esclarecimento.

Foram incluídas mulheres sexualmente ativas com experiência orgástica individual ou com o parceiro, pelo menos um parto de nascituro vivo; parceiro atual fixo e capacitado para o coito, e que, após a histerectomia, permanecessem com pelo menos um dos ovários. Foram excluídas as mulheres portadoras de neoplasia maligna genital ou extragenital; com doenças sistêmicas graves; endocrinopatias; antecedentes ou acometidas de doenças psiquiátricas, distúrbios de cognição, ou em uso de medicamentos que pudessem interferir diretamente na resposta sexual.

Do total de mulheres selecionadas, 48 satisfizeram os critérios de inclusão e exclusão no início do estudo. Deste total, somente 33 foram encontradas e retornaram para a segunda avaliação após seis meses.

Após entrevista inicial, todas as pacientes foram arguidas por um mesmo examinador e submetidas a dois instrumentos para avaliação da sexualidade, a saber: Quociente Sexual - Versão Feminina (QS-F) e Inventário de Satisfação Sexual - Versão Feminina 
(GRISS). Os mesmos instrumentos foram aplicados pelo mesmo examinador seis meses após o procedimento.

O QS-F foi desenvolvido no Brasil e leva em conta os vários domínios da função sexual feminina, sendo constituído por um conjunto de perguntas individuais delineadas para aferir fenômenos específicos chamados "construtos", que, no caso desta pesquisa, é a função sexual.

O Inventário de GRISS (Golombok-Rust Inventory of Sexual Satisfaction ${ }^{14}$ ) é composto por 28 perguntas que avaliam a resposta sexual e o relacionamento com o parceiro. As respostas variam de acordo com a intensidade, de nenhuma até a mais alta intensidade observada ou experimentada pela mulher, em relação ao questionamento realizado em cada tópico do instrumento de avaliação. Cada avaliação recebe uma nota ou escore que varia de 0 a 9 e que representa a frequência dos eventos abordados (nunca; quase nunca; ocasionalmente; geralmente; sempre).

Estes instrumentos possibilitam a obtenção de informações sobre a maneira como cada mulher se relaciona com o próprio corpo e com o corpo do seu parceiro, o nível de interesse sexual, a qualidade de resposta à excitação, da frequência da atividade sexual e da obtenção do orgasmo. A aplicação desenvolveuse nos dois momentos (antes e após seis meses da histerectomia) de forma individual e pelo mesmo pesquisador. Seus resultados também foram analisados e interpretados à luz do referencial teórico da psicologia sócio-histórica - esta teoria do conhecimento está associada à compreensão da estrutura da cultura, à organização social e ao resgate da subjetividade humana.

As variáveis quantitativas (GRISS) foram avaliadas pelo teste de Wilcoxon e as qualitativas (QS-F) pelo teste do $\chi^{2}$. Foram considerados valores de $\mathrm{p}<0,05$ para comprovar diferença significante. As análises foram feitas pelos programas Epi-Info for Windows versão 3.3.2 e Statistical Package for Social Sciences versão 13.0 for Windows.
Resultados

A média de idade ( \pm desvio padrão) das 33 mulheres incluídas neste estudo foi de 44,2 $\pm 4,3$ anos. Desse total, $24(72,7 \%)$ reportaram união estável com relacionamento amoroso classificado como "bom" ou "ótimo" e 29 (87,9\%) com relacionamento sexual "bom" ou "ótimo". Dados complementares estão descritos na Tabela 1.

Pelo QS-F, notou-se que $13(39,4 \%)$ pacientes exibiram piora no relacionamento sexual; $11(33,3 \%)$ melhoraram e a histerectomia não exerceu impacto positivo ou negativo em $9(27,3 \%)$ pacientes. Não foi notada associação entre os resultados obtidos no QS-F antes e depois da histerectomia $\left(\chi^{2}=10,6\right.$; grau de liberdade $=12 ; \mathrm{p}=0,05$, Tabela 2).

Os escores médios obtidos após a aplicação do questionário de GRISS mostraram piora significante nos parâmetros "satisfação sexual" ( $\mathrm{p}=0,03)$; "expressão da sensualidade feminina" ( $\mathrm{p}=0,01)$; "vaginismo/dispareunia" $(\mathrm{p}=0,02)$ e "anorgasmia" $(\mathrm{p}=0,04)$. A média dos escores obtidos nos demais aspectos abordados ("frequência das relações sexuais", "comunicação sexual" e "evitação sexual feminina") antes do procedimento cirúrgico não apresentou diferença significante quando comparada àquela obtida antes da histerectomia. A Tabela 3 mostra as medidas e a comparação dos dados obtidos pelo instrumento GRISS.

Tabela 1 - Antecedentes obstétricos e ginecológicos das 33 pacientes incluídas no estudo

\begin{tabular}{lc}
\hline Variáveis & Média ( \pm desvio padrão) \\
\hline Idade na menarca & $13,4 \pm 2,2$ \\
Número de gestações & $3,0 \pm 1,5$ \\
Número de parłos & $2,6 \pm 1,2$ \\
Número de fillhos & $2,5 \pm 1,1$ \\
Idade na primeira relação sexual & $19,1 \pm 2,8$ \\
Idade no primeiro orgasmo & $19,7 \pm 2,8$ \\
\hline
\end{tabular}

Tabela 2 - Avaliação do Quociente Sexual - Versão feminina (QS-F) antes e após a histerectomia

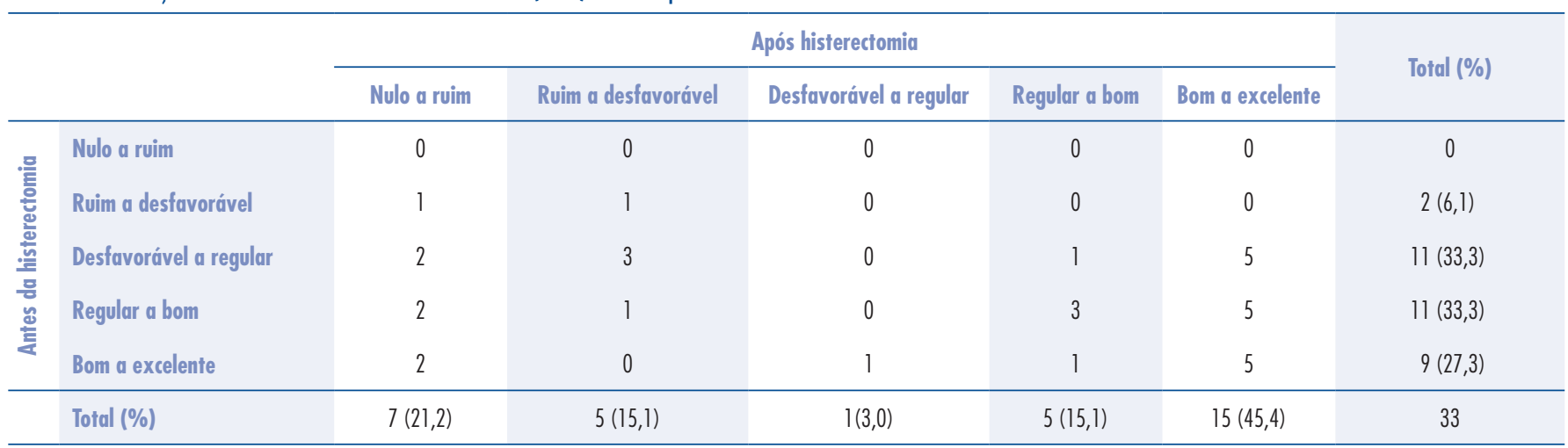

$\chi^{2}=10,69 ;$ grau de liberdade $=2 ; p=0,55$. 
Tabela 3 - Distribuição dos escores obtidos após a aplicação do inventário de GRISS antes e após histerectomia (teste de Wilcoxon)

\begin{tabular}{|c|c|c|c|}
\hline & & Média \pm desvio padrão & Valor de $\mathrm{p}$ \\
\hline \multirow[t]{2}{*}{ Frequência de relações sexuais } & Antes & $5,1 \pm 2,5$ & 0,5 \\
\hline & Após & $4,8 \pm 3,5$ & \\
\hline \multirow{2}{*}{ Comunicação sexual } & Antes & $4,7 \pm 3,1$ & 0,1 \\
\hline & Após & $5,4 \pm 3,0$ & \\
\hline \multirow[t]{2}{*}{ Satisfação sexual } & Antes & $2,7 \pm 2,1$ & 0,03 \\
\hline & Após & $3,6 \pm 2,9$ & \\
\hline \multirow[t]{2}{*}{ Evifação sexual feminina } & Antes & $4,4 \pm 2,2$ & 0,6 \\
\hline & Após & $4,7 \pm 3,6$ & \\
\hline \multirow[t]{2}{*}{ Expressão de sensualidade feminina } & Antes & $2,6 \pm 2,1$ & 0,01 \\
\hline & Após & $3,9 \pm 3,4$ & \\
\hline \multirow[t]{2}{*}{ Vaginismo/dispareunia } & Antes & $2,1 \pm 1,5$ & 0,02 \\
\hline & Após & $3,4 \pm 2,9$ & \\
\hline \multirow[t]{2}{*}{ Anorgasmia } & Antes & $2,9 \pm 1,3$ & 0,04 \\
\hline & Após & $4,2 \pm 3,0$ & \\
\hline
\end{tabular}

\section{Discussão}

Para que sejam feitas aferições clínicas, como a função sexual, por exemplo, não há padrões físicos de validade, e foi por conta disso que, neste estudo, optou-se pelo emprego de instrumentos validados, como o QS-F e o GRISS, que fornecem resultados predominantemente qualitativos. Nesse sentido, apesar de sua natureza qualitativa e de algumas limitações de generalização deste trabalho, foi possível notar que é iminente a necessidade de discutir o impacto da retirada do útero para a elaboração da autoimagem feminina com consequente reconfiguração social e, principalmente, sexual.

Os resultados apresentados indicam que as mulheres a serem submetidas à histerectomia podem apresentar comprometimento na sexualidade, reprodução e à função sexual. Para elas, a histerectomia poderia ser o evento que, de fato, encerraria o período reprodutivo de sua vida com grandes comprometimentos na sua sexualidade. Tal associação entre reprodução e sexualidade tem sido abordada na literatura em várias áreas do conhecimento e direcionada à compreensão de como as mulheres vivenciam essas mudanças em sua condição reprodutiva ${ }^{6,15}$. Apesar de as mulheres sentirem-se aliviadas com a possibilidade do desaparecimento dos sintomas incômodos da doença, apresentam mitos e crendices com a retirada do útero, relacionados à sua identidade social e de gênero, à falta de conhecimento de seu corpo, dos órgãos e suas respectivas funções ${ }^{7}$.

Neste estudo, ficou evidenciado que mais da metade das mulheres apresentava incertezas em relação à retirada do útero e sobre o real impacto deste procedimento sobre a vida sexual, assim como também reportavam sentimentos de tristeza por perderem um órgão importante contrapostos ao desejo de cura. É sabido que a sexualidade se sobrepõe aos limites do impulso genital, sendo este apenas um dos elementos da relação sexual em que intervêm.

Para o bom exercício da sexualidade, compreendemse fatores como a afetividade, a fantasia, a emoção, a comunicação e o tipo de estímulo sensorial individual e particular mais efetivo na obtenção do desejo e do prazer. Estudo $^{16}$ aponta que a falta de esclarecimentos e orientações por parte dos profissionais de Saúde, combinada à grave carência de informações das mulheres a respeito do funcionamento dos órgãos genitais (associam a capacidade sexual à presença do útero), pode desencadear inúmeros problemas de ordem emocional, como: gerar sentimentos de culpa, medo, vergonha, insegurança, agitação, além de depressão e insônia; comprometer a imagem corporal, entre outros.

Acredita-se que algumas medidas profiláticas poderão ser tomadas em relação às disfunções sexuais, tais como a informação das portadoras de leiomioma uterino sobre a possibilidade da histerectomia logo no início da doença, ressaltando a necessidade da compreensão da anatomia e do funcionamento do próprio corpo, incluindo-se orientação e aconselhamento especializado e objetivando esclarecimento de ideias errôneas, preconceituosas e deficitárias a respeito do exercício da sexualidade ${ }^{17}$.

É importante o preparo dos profissionais de Saúde (médicos, enfermeiros, fisioterapeutas, psicólogos, entre outros) para o conhecimento e manejo técnico dos problemas que envolvem questões sexuais, tendo em vista que estes profissionais recebem treinamento insuficiente ou nulo na sua formação ${ }^{18}$.

Essa discussão caminha para a importância da contribuição dos profissionais de Saúde tomando conhecimento da qualidade de vida sexual, sobretudo na mulher com queixa de leiomioma do útero, com risco iminente da histerectomia ${ }^{19,20}$ de adotar medidas preventivas quanto ao exercício de sua função sexual futura.

Sem dúvida, a criação de um protocolo de atendimento pré-operatório padronizado para mulheres, com informações esclarecedoras sobre a histerectomia, associado ao significado do funcionamento dos seus órgãos genitais e à relação que estabelece o papel da feminilidade, possibilitaria a compreensão sobre a sexualidade feminina e auxiliaria na manutenção de uma prática sexual mais saudável antes e após a intervenção cirúrgica.

É relevante pensar que o atendimento interdisciplinar da mulher, ajudaria a refletir não somente sobre a identidade feminina, mas na esfera mais ampla da saúde $^{21}$. Parte dos abalos emocionais e sexuais vivenciados por elas, tanto no período que antecede quanto após o procedimento cirúrgico, poderiam ser minimizados, caso 
fossem acompanhadas desde o momento que recebem a noticia da doença ${ }^{22-24}$.

Em conclusão, a histerectomia parece prejudicar a vida sexual e a sexualidade das mulheres, pois o útero está diretamente associado a estas funções. Acredita- se que um atendimento psicológico e um ambiente hospitalar favorável parecem ser imprescindíveis para que as mulheres possam vivenciar e elaborar as repercussões da retirada do útero de maneira menos prejudicial possível.

\section{Referências}

1. Duarte G. Doenças benignas do corpo do útero. In: Halbe HW. Tratado de ginecologia. 3a ed. São Paulo: Roca; 2000. p. 1273 300.

2. Lemgruber I, Lemgruber M. Histerectomias. In: Oliveira HC, Lemgruber I, Costa OT, editores. Tratado de ginecologia FEBRASGO. Rio Janeiro: Revinter; 2000. p. 1383-401.

3. Stovall TG. Histerectomia. In: Berek JS, editor. Novak tratado de ginecologia. 13a ed. Rio de Janeiro: Guanabara Koogan; 2002. p. 708-45.

4. Naughton M, McBee WL. Health-related quality of life after hysterectomy. Clin Obstet Gynecol. 1997;40(4):947-57.

5. Caliri MHL, Cunha AMP. A experiência da mulher ao enfrentar a histerectomia. Femina. 1998;26(9):749-52.

6. Novoa AM. Histerectomia: efeitos emocionais na identidade feminina. Reprodução. 1991;6(4):193-5.

7. Sbroggio AMR, Osis MMD, Bedone AJ. O significado da retirada do útero para as mulheres: um estudo qualitativo. Rev Assoc Med Bras. 2005;51(5):270-4.

8. Cavalcanti AL, Bagnoli VR, Fonseca AM, Fegies L, Pinotti JA Sexualidade nas mulheres histerectomizadas. Rev Ginecol Obstet. 2002;13(3):171-84.

9. Gonçalves N. Distúrbios ginecológicos e sexualidade. In: Piato S, editor. Tratado de ginecologia. São Paulo: Artes Médicas; 2002. p. 944-7.

10. Jewett JG. Vaginal length and incidence of dyspareunia following total abdominal hysterectomy. Am J Obstet Gynecol. 1952:63(2):400-7.

11. Zobbe V, Gimbel H, Andersen BM, Filtenborg T, Jakobsen K, Sørensen HC, et al. Sexuality after total vs. subtotal hysterectomy. Acta Obstet Gynecol Scand. 2004;83(2):191-6.

12. Goetsch MF. The effect of total hysterectomy on specific sexual sensations. Am J Obstet Gynecol. 2005;192(6): 1922-7.

13. Roovers JP, van der Bom JG, van der Vaart $\mathrm{CH}$, Heintz AP. Hysterectomy and sexual wellbeing: prospective observational study of vaginal hysterectomy, subtotal abdominal hysterectomy, and total abdominal hysterectomy. BM. 2003;327(7418):744-8

14. Golombok S, Rust J. The Golombok and Rust inventory of sexual satisfaction. London: NFER-Nelson; 1986.

15. Anker LW. Vivências psicológicas associados à histerectomia: um enfoque psicanalítico [dissertação]. São Paulo: Pontifícia Universidade Católica; 1990.

16. Urrutia SMT, Araya G A, Villarroel del PL, Viñales AD. Características y evolución de la sexualidad en mujeres histerectomizadas. Rev Chil Obstet Ginecol. 2004;69(4):301-6.

17. Rhodes JC, Kjerulff KH, Langenberg PW, Guzinski GM. Hysterectomy and sexual functioning. JAMA. 1999;282(20):1934-41.

18. Lowenstein L, Yarnitsky D, Gruenwald I, Deutsch M, Sprecher E, Gedalia U, et al. Does hysterectomy affect genital sensation? Eur J Obstet Gynecol Reprod Biol. 2005; 1 19(2):242-5.

19. Roussis NP, Waltrous L, Kerr A, Robertazzi R, Cabbad MF. Sexual response in the patient after hysterectomy: total abdominal versus supracervical versus vaginal procedure. Am J Obstet Gynecol. 2004; 190(5): 1427-8.

20. Kim DH, Lee YS, Lee ES. Alteration of sexual function after classic intrafascial supracervical hysterectomy and total hysterectomy. J Am Assoc Gynecol Laparosc. 2003;10(1):60-4.

21. Jewett JG. Vaginal length and incidence of dyspareunia following total abdominal hysterectomy. Am J Obstet Gynecol. 1952;63(2):400-7.

22. Cavalcanti R, Cavalcanti M. Tratamento clínico das inadequações sexuais. 3a ed. São Paulo: Roca; 2006.

23. Gonçalves N. Disfunções sexuais. In: Piato S, editor. Terapêutica endócrina em ginecologia. São Paulo: Artes Médicas; 2005. p. 193-8.

24. Gonçalves N. A abordagem da sexualidade na consulta ginecológica. In: Etiene MA, Waitman MC, editores. Disfunções sexuais femininas: a fisioterapia como recurso terapêutico. São Paulo: LMP; 2006. p. 153-6. 